\title{
Novel Techniques for Constraining Neutron-Capture Rates Relevant to Heavy-element Nucleosynthesis
}

\author{
A. C. Larsen ${ }^{* a}$, A. Spyrou ${ }^{b, c, d}$, S. N. Liddick ${ }^{b, e}$, M. Guttormsen $^{a}$, V. W. Ingeberg ${ }^{a}$, \\ S. Siem ${ }^{a}$, M. Wiedeking ${ }^{f}$, B. P. Crider ${ }^{b}$, F. Naqvi ${ }^{g}$, G. Perdikakis ${ }^{h, b, d}$, \\ M. R. Mumpower ${ }^{j}$, R. Surman ${ }^{g}$, G. M. Tveten ${ }^{a}$, D. L. Bleuel ${ }^{i}$, A. Couture ${ }^{j}$, \\ L. Crespo Campo ${ }^{a}$, A. C. Dombos ${ }^{b, c, d}$, A. Görgen ${ }^{a}$, R. Lewis ${ }^{b, e}$, S. M. Mosby ${ }^{j}$, \\ S. Nikas ${ }^{h}$, C. J. Prokop ${ }^{b, e, j}$, S. J. Quinn ${ }^{b, c, d}$, T. Renstrøm ${ }^{a}$, and B. Rubio ${ }^{k}$ \\ ${ }^{a}$ Department of Physics, University of Oslo, N-0316 Oslo, Norway \\ ${ }^{b}$ National Superconducting Cyclotron Laboratory (NSCL), Michigan State University, \\ East Lansing, Michigan 48824, USA \\ ${ }^{c}$ Department of Physics and Astronomy, Michigan State University, \\ East Lansing, Michigan 48824, USA \\ ${ }^{d}$ Joint Institute for Nuclear Astrophysics, Michigan State University, \\ East Lansing, Michigan 48824, USA \\ ${ }^{e}$ Department of Chemistry, Michigan State University, East Lansing, Michigan 48824, USA \\ $f$ iThemba LABS, 7129 Somerset West, South Africa \\ ${ }^{g}$ Department of Physics, University of Notre Dame, Notre Dame, IN 46556-5670, USA \\ ${ }^{h}$ Central Michigan University, Mount Pleasant, Michigan 48859, USA \\ ${ }^{i}$ Lawrence Livermore National Laboratory, Livermore, California 94550-9234, USA \\ j Los Alamos National Laboratory, Los Alamos, New Mexico 87545, USA \\ ${ }^{k}$ IFIC, CSIC-Universidad de Valencia, Spain \\ E-mail: a.c.larsen@fys.uio.no
}

\begin{abstract}
In this contribution we discuss new experimental approaches to indirectly provide information on neutron-capture rates relevant to the $r$-process. In particular, we focus on applications of the Oslo method to extract fundamental nuclear properties for reaction-rate calculations: the nuclear level density and the $\gamma$ strength function. Two methods are discussed in detail, the Oslo method in inverse kinematics and the beta-Oslo method. These methods present a first step towards constraining neutron-capture rates of importance to the $r$-process.
\end{abstract}

The 26th International Nuclear Physics Conference

11-16 September, 2016

Adelaide, Australia

* Speaker. 


\section{Introduction}

One of the big mysteries mankind has pondered, is how and where the elements observed in the Universe were formed. The first attempt to determine the distribution of element abundances was made by Goldschmidt in 1937 [1], and has later been substantially improved with precise measurements of CI1 carbonaceous chondrites, terrestrial samples, and analysis of solar spectra [2]. In 1957, Burbidge, Burbidge, Fowler and Hoyle [3] and Cameron [4] outlined the main nucleosynthesis processes called for to explain the observed abundances. However, 60 years later, we still do not fully understand how the heavy elements (above iron, $Z=26$ ) were created.

Amongst the processes responsible for the heavy-element nucleosynthesis, the rapid neutroncapture process ( $r$-process) is perhaps the most challenging one to describe, both from an astrophysics and nuclear-physics point of view. This process accounts for about $50 \%$ of the heavy elements and is the only process able to produce actinides [5]. We know that the $r$-process is operating under extreme neutron densities $\left(\sim 10^{20} / \mathrm{cm}^{3}\right)$ and short time scales (seconds). Traditionally, these conditions were believed to be achieved during a core-collapse supernova. This picture is blurred by recent state-of-the-art supernova simulations, indicating that neither the necessary neutron flux nor the entropy required are available-not to mention the failure of making the simulated core-collapse supernova explode [6,7]. Another astrophysical candidate that has grown rapidly in popularity is the merging system of two neutron stars, which can produce a quite robust solar-system-like abundance pattern for $A>130[8,9]$. With the advance in detecting gravitational waves with LIGO [10], and one possible measurement of the $r$-process elements' afterglow ("kilonova") [11, 12], some cautious optimism of expecting a first-ever direct $r$-process observation in the near future might be in order.

Astrophysics aside, the enormous amount of needed nuclear-data input is quite overwhelming. The $r$-process undoubtedly involves highly exotic, neutron-rich nuclei, for which the existence of many of them are not yet confirmed experimentally. For a state-of-the-art $r$-process reaction network, as many as $\approx 5000$ nuclei and $\approx 50,000$ reaction rates must be taken into account. Clearly, many of these reaction rates will have to be estimated theoretically. Today's standard approach is to use nuclear-reaction codes for calculating the compound-nucleus cross-section contribution through the Hauser-Feshbach formalism [13], and also often adding various non-statistical effects such as pre-equilibrium processes (e.g. [14]), direct capture (e.g. [15]) and resonance capture [16]. Nonetheless, even for nuclei near the valley of stability, theoretical cross-section predictions vary by a factor $\sim 5-10$, and for very exotic nuclei the uncertainty in e.g. neutron-capture rates can reach several orders of magnitude. In this context, sensitivity studies such as Refs. [17, 18, 19] are very useful for identifying specific cases that are most important for the final abundances.

Here we discuss new approaches to experimentally constrain radiative neutron-capture rates for neutron-rich nuclei. As direct $(n, \gamma)$ cross-section measurement on such short-lived nuclei are out of reach at the moment, and will be so for many years to come, we concentrate on indirect methods where the basic ingredients entering the cross-section calculations are measured.

\section{Input for cross-section and reaction-rate calculations-the Oslo method}

To calculate astrophysical, Maxwellian-averaged reaction rates, one usually assumes ther- 
modynamic equilibrium for both the target nucleus and the projectile, thus obeying MaxwellBoltzmann distributions for a given temperature $T$ at the specific stellar environment. Because of the temperature at the astrophysical site, the target nucleus might well be in an excited state, which will contribute to the rate. Specifically, the $(n, \gamma)$ reaction rate $N_{A}\langle\sigma v\rangle_{n \gamma}$ is found from integrating the cross section over a Maxwell-Boltzmann distribution of energies $E$ at a given $T$ :

$$
N_{A}\langle\sigma v\rangle_{n \gamma}(T) \propto \int_{0}^{\infty} \sum_{\mu} \frac{2 I^{\mu}+1}{2 I^{0}+1} \sigma_{n \gamma}^{\mu}(E) E \exp \left[-\frac{E+E_{x}^{\mu}}{k_{B} T}\right] \mathrm{d} E .
$$

Here, $N_{A}$ is Avogardo's number, $\mu$ denotes the excited state, $I^{0}$ and $I^{\mu}$ are the spin of the ground state and excited states, respectively, $E$ is the relative energy of the neutron and target, $E_{x}^{\mu}$ is the excitation energy for the state $\mu$, and $k_{B}$ is Boltzmann's constant. We see from this expression that the reaction rate is proportional to the cross section $\sigma_{n \gamma}$. Hence, to estimate a correct reaction rate, it is crucial to determine the cross section.

For radiative neutron capture, the main ingredients determining the cross section are the nuclear level density (NLD) and the $\gamma$ strength function $(\gamma \mathrm{SF})$ of the residual nucleus, and the neutron optical-model potential for the target nucleus, i.e. $\sigma_{n \gamma} \propto \mathscr{T}_{n}\left(E_{n}\right) \rho\left(E_{n}+S_{n}\right) \mathscr{T}\left(E_{\gamma}\right)$ where $\mathscr{T}_{n}$ is the neutron transmission coefficient for the target-plus-neutron system determined by the neutron optical-model potential (n-OMP), $\rho\left(E_{n}+S_{n}\right)$ is the NLD in the compound system at the neutron separation energy $S_{n}$ plus the incoming neutron energy, and $\mathscr{T}\left(E_{\gamma}\right)$ is the $\gamma$-ray transmission coefficient directly proportional to the $\gamma \mathrm{SF}$. The NLD is a measure of the available quantum levels, and is simply defined as $\rho\left(E_{x}\right)=\Delta N\left(E_{x}\right) / \Delta E_{x}$, where $\Delta N\left(E_{x}\right)$ is the number of levels at an excitation energy $E_{x}$ for an energy bin $\Delta E_{x}$. The $\gamma \mathrm{SF}$ gives information on the average reduced decay width for a given $\gamma$-transition energy $E_{\gamma}$ of a particular multipole type $L$ and electromagnetic character $X$ [23]: $f_{X L}\left(E_{\gamma}\right)=\mathscr{T}\left(E_{\gamma}\right) / 2 \pi E_{\gamma}^{(2 L+1)}=\left\langle\Gamma_{X L}\right\rangle \rho\left(E_{i}\right) / E_{\gamma}^{(2 L+1)}$, where $E_{i}$ denotes the initial excitation energy.

Both the NLD and $\gamma \mathrm{SF}$ can be measured in one and the same experiment with the wellestablished Oslo method [20, 21, 22]. The main principles behind this method are:

1. measure $\gamma$-ray spectra as function of initial excitation energy $E_{i}$;

2. correct the $\gamma$ spectra for the detector response [21];

3. extract the distribution of the $\gamma$ rays emitted first in all decay cascades (primary $\gamma$ rays) for a given $E_{i}[22]$;

4. perform a simultaneous fit of all primary $\gamma$-ray spectra for a selected $E_{i}$ range, and obtain the NLD and $\gamma$ SF [20];

5. normalize the NLD and $\gamma$ SF and evaluate systematic errors [20,24].

One of the most surprising discoveries with the Oslo method is the upbend, an unexpected increase in the $\gamma \mathrm{SF}$ at low transitions energies and high excitation energies. This peculiar feature was first seen in Fe isotopes [25], and later on found in many nuclei such as ${ }^{43-45} \mathrm{Sc}$ [26, 27], ${ }^{93-98} \mathrm{Mo}$ [28], ${ }^{138,139} \mathrm{La}$ [29] and very recently in the heavy ${ }^{151,153} \mathrm{Sm}$ [30]. The upbend was independently confirmed in ${ }^{95} \mathrm{Mo}$ using a completely different technique to map out the functional 
form of the $\gamma \mathrm{SF}$ [31], and it was shown to be dominantly of dipole nature in ${ }^{56} \mathrm{Fe}$ [32]. At present, it is not clear whether it is of electric [33] or magnetic type [34,35]. If the upbend turns out to be present also for very neutron-rich nuclei, this could boost the $(n, \gamma)$ reaction rates by $\sim 2$ orders of magnitude [36]. To prove or disprove this, one must measure the $\gamma \mathrm{SF}$ of neutron-rich nuclei.

In recent publications [37, 38, 39, 40, 41, 42], the NLD and $\gamma$ SF measured with the Oslo method have been successfully used as input to the TALYS nuclear reaction code $[43,44]$ to calculate radiative capture cross-sections. However, the standard Oslo method is limited to nuclei close to the valley of stability. In the following, we describe new approaches to extract the NLD and $\gamma \mathrm{SF}$ for neutron-rich nuclei.

\section{New experimental techniques}

Here we sketch the main principles of two new experimental approaches to measure NLD and $\gamma \mathrm{SF}$ for exotic nuclei. Both approaches use the basic elements of the standard Oslo method, but have different experimental features to obtain the starting point for the NLD and $\gamma$ SF extraction, namely $\gamma$-ray spectra as function of initial excitation energy $E_{i}$. Also the two new methods encounter different challenges as will be briefly discussed.

\subsection{The Oslo method in inverse kinematics}

The standard Oslo method employs a light-ion beam impinging on a heavy target, measuring the charged ejectiles in coincidence with the emitted $\gamma$ rays. Hence, the energy of the charged ejectiles gives information on $E_{i}$ for the residual nucleus (below $S_{n}$ ), and the $\gamma$ rays are tagged with that specific initial excitation energy. Typically, one needs $\approx 40,000$ particle- $\gamma$ coincidences to get reasonable error bars on the NLD and $\gamma \mathrm{SF}$.

At radioactive-beam facilities such as e.g. HIE-ISOLDE [45], a heavy, exotic nucleus is produced, separated from the other nuclear species created and re-accelerated to a secondary target where it can undergo nuclear reactions. The secondary target would now contain light elements, e.g. deuterium, and the reaction is inversed compared to the heavy-target-light-beam situation. Now, one would measure the light target-like particle with segmented silicon detectors, and the subsequent $\gamma$ rays with a highly efficient $\gamma$-ray detector array. Provided sufficient intensity on the radioactive (heavy) beam and a decent cross section on the desired reaction, e.g. $(d, p \gamma)$, one would then obtain a similar set of coincidence data as for the standard Oslo method.

The first proof-of-principle experiment demonstrating the feasibility of the Oslo method in inverse kinematics, was performed at iThemba LABS in 2015 [46], with a $300-\mathrm{MeV}^{86} \mathrm{Kr}$ beam directed onto $\mathrm{C}_{2} \mathrm{D}_{4}$ and $\mathrm{C}_{8} \mathrm{H}_{3} \mathrm{D}_{5}$ targets. Charged particles were measured with two DSSD detectors in a $\Delta E-E$ configuration, while the $\gamma$ rays were detected with the AFRODITE array comprised of eight BGO-shielded CLOVER detectors and two large-volume $\mathrm{LaBr}_{3}(\mathrm{Ce})$ detectors. Data from the ${ }^{86} \mathrm{Kr}(d, p \gamma){ }^{87} \mathrm{Kr}$ reaction was selected and the NLD and $\gamma \mathrm{SF}$ was extracted from the coincidence data, showing very promising results and excellent agreement with previous data from other measurements [47].

Further, the first Oslo-method inverse-kinematics experiment on a radioactive, neutron-rich nucleus was completed at HIE-ISOLDE, CERN, in November 2016 [48], utilizing a $\approx 4.5-\mathrm{MeV} /$ nucleon 
${ }^{66} \mathrm{Ni}$ beam impinging on a $\mathrm{C}_{2} \mathrm{D}_{4}$ target. Particles from the ${ }^{66} \mathrm{Ni}(d, p \gamma){ }^{67} \mathrm{Ni}$ and ${ }^{66} \mathrm{Ni}(d, t \gamma){ }^{65} \mathrm{Ni}$ reactions were collected, using C-REX (an upgrade of the T-REX detector array [49]) for chargedparticle detection. The $\gamma$ rays were measured with MINIBALL [50] and in addition six largevolume $\mathrm{LaBr}_{3}(\mathrm{Ce})$ detectors. The run was very successful and the data are currently being analyzed.

\subsection{The beta-Oslo method}

In many cases it is hard to achieve an intense enough radioactive beam to apply the Oslo method in inverse kinematics. One of the main strengths with the recently invented beta-Oslo method [51] is that one can obtain sufficient statistics with an implantation rate of 1 particle per second. Here, one makes use of the fact that for neutron-rich nuclei, the $Q$-value for beta decay is comparable to or even higher than the neutron separation energy, and so will populate excited states in a broad energy range in the daughter nucleus. Further, using a segmented total-absorption spectrometer such as the SuN detector [52], one gets the sum of all $\gamma$ rays in the cascades that is equivalent to the initial excitation energy, while the single segments give the individual $\gamma$ rays. Thus, one can obtain a set of excitation-energy tagged $\gamma$-ray spectra and apply the Oslo method to extract NLD and $\gamma \mathrm{SF}$ for the daughter nucleus.

The beta-Oslo method was first applied on ${ }^{76} \mathrm{Ga}$ beta-decaying into ${ }^{76} \mathrm{Ge}$ [51]. The experiment was performed at the National Superconducting Cyclotron Laboratory (NSCL), Michigan State University (MSU), using a $130-\mathrm{MeV} /$ nucleon ${ }^{76} \mathrm{Ge}$ beam producing ${ }^{76} \mathrm{Ga}$ by fragmentation on a thick beryllium target. The ${ }^{76} \mathrm{Ga}$ secondary beam was first guided through the A1900 fragment separator [53], and then thermalized in the large-volume gas cell [54] and delivered to the
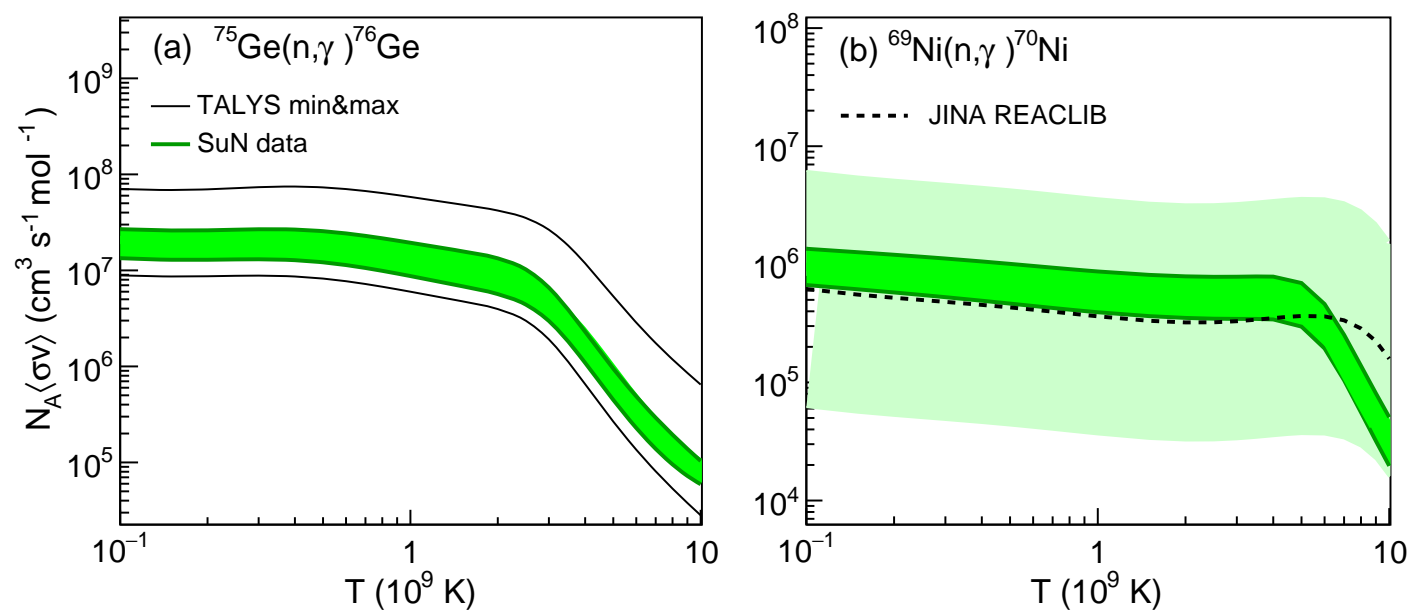

Figure 1: (Color online) Radiative neutron-capture reaction rates for (a) ${ }^{75}(n, \gamma){ }^{76} \mathrm{Ge}$ [51] and (b) ${ }^{69} \mathrm{Ni}(n, \gamma){ }^{70} \mathrm{Ni}[55]$. In panel a, we compare with the intrinsic uncertainty obtained by varying all NLD and $\gamma \mathrm{SF}$ models in TALYS-1.6 (black solid lines). The data-constrained rate is shown in the green band. In panel b, we follow Ref. [18] and compare with the JINA REACLIB rate [57] (dashed line) scaled with a factor of 10 up and down (light-green band). 
experimental setup, where the ${ }^{76} \mathrm{Ga}$ beam was implanted on an SSB detector mounted inside SuN, detecting only the beta particles due to the low beam energy $(\approx 30 \mathrm{keV}$ after the gas cell). SuN was then detecting the subsequent $\gamma$-ray cascades in the daughter nucleus ${ }^{76} \mathrm{Ge}$. Although ${ }^{76} \mathrm{Ge}$ is the most neutron-rich, stable Ge isotope in nature, the uncertainty of the ${ }^{75} \mathrm{Ge}(n, \gamma){ }^{76} \mathrm{Ge}$ reaction rate obtained by varying all NLD, $\gamma \mathrm{SF}$ and $\mathrm{n}$-OMP models in TALYS is a factor of $\approx 8$. As shown in Fig. 1a, using the SuN data as input in TALYS, the uncertainty was significantly reduced to about a factor of $\approx 2$.

Further, the beta-Oslo method has very recently been applied on the neutron-rich ${ }^{70}$ Co betadecaying into ${ }^{70} \mathrm{Ni}$ [55]. This experiment was performed at NSCL, MSU, where a primary 140$\mathrm{MeV} /$ nucleon ${ }^{86} \mathrm{Kr}$ beam hit a beryllium target to produce ${ }^{70} \mathrm{Co}$ through fragmentation. The fragmentation reaction products were separated with the A1900 separator and delivered to the experimental setup, this time with a DSSD inside SuN, detecting both the fragment and the beta particle. Again, SuN was used to detect the $\gamma$-ray cascades from the daughter nucleus, ${ }^{70} \mathrm{Ni}$. Complementary data from GSI on the ${ }^{68} \mathrm{Ni} \gamma \mathrm{SF}$ [56] above the neutron separation energy allowed for a well-determined absolute normalization of the full $\gamma \mathrm{SF}$, giving uncertainties down to a factor $2-3$ in the deduced ${ }^{69} \mathrm{Ni}(n, \gamma){ }^{70} \mathrm{Ni}$ reaction rate (see Fig. 1b). This is to be compared with the uncertainty band considered in Ref. [18] of a factor of 100 (multiplying the JINA REACLIB rate [57] with a factor 0.1 and 10). It is clear that the data-constrained rates represent a significant improvement. As shown in Ref. [55], the rates need to be determined within at least a factor of 10 to be able to meaningfully compare fine structures in the isotopic abundances with the nucleosynthesis simulation.

\section{Challenges and future prospects}

For both the new methods highlighted here, absolute normalization of the results is challenging, and new methods for normalization are currently in progress.

For the Oslo method in inverse kinematics, the main challenge is achieving high enough intensities for the radioactive beam of interest, limiting the range of nuclei that can be reached at present facilities. Also, obtaining sufficient excitation-energy resolution (well below $1 \mathrm{MeV}$ FWHM) might be difficult depending on the case and the reaction kinematics.

For the beta-Oslo method, one needs to take into account the rather narrow spin range that is populated through beta decay in the daughter nucleus. This is rather straight-forward as long as the spin of the mother nucleus is known, but this might not always be the case for very exotic nuclei. Also, the determination of the initial excitation energy might suffer from incomplete summing, making a low-energy tail towards lower $E_{i}$. This effect is typically small for the highly efficient $\mathrm{SuN}$ detector [52]. Further improvement would include correcting for incomplete summing on the excitation energy in addition to the $\gamma$ energy; the latter is already done and the former is work in progress. Finally, in cases where the beta-decay $Q$-value is very high (several $\mathrm{MeV}$ above $S_{n}$ ), the $\beta-n$ channel could be a significant decay mode. At present, it is not possible to discriminate against neutrons in SuN; it would be highly desirable to implement that opportunity in future TAS detector designs.

Regarding future prospects, and considering the huge achievements both in nuclear-physics experiments and nuclear theory, as well as in astronomy observations and models, we are now at 
a very exciting point in history. New radioactive-beam facilities such as FRIB [58] and FAIR [59] will greatly extend the experimental reach of exotic nuclei, providing the ultimate testing ground for nuclear theory and enabling many more $(n, \gamma)$ reaction rates to be experimentally constrained, amongst other experimental information such as beta-decay rates and nuclear masses. This will in turn significantly improve the input for $r$-process nucleosynthesis simulations, limiting the parameter space which is at present very large. Also, with the rise of even more sensitive gravitationalwave detectors such as advanced LIGO [60] to measure neutron-star mergers, combined with an electromagnetic follow-up to detect $\gamma$-ray bursts and the kilonova, (e.g. Ref. [61]) the nuclearastrophysics community might see the day where the $r$-process astrophysical site is finally confirmed.

\section{Acknowledgments}

We gratefully acknowledge the support of NSCL operations staff. A. C. L. gratefully acknowledges funding through ERC-STG-2014 under grant agreement no. 637686. S. S. and G. M. T. acknowledge financial support by the NFR under project grant no. 210007 and 222287, respectively. M. W. acknowledges support by the National Research Foundation of South Africa under grant no. 92789, and by the DST and SA-CERN of South Africa. D. L. B. acknowledges the support of LLNL under Contract DE-AC52-07NA27344. The LANL work was carried out under the auspices of the NNSA of the U.S. Department of Energy at Los Alamos National Laboratory under Contract No. DE-AC52-06NA25396. This work was supported by the National Science Foundation under Grants No. PHY 1102511 (NSCL) and No. PHY 1430152 (Joint Institute for Nuclear Astrophysics), and PHY 1350234 (CAREER). This material is based upon work supported by the Department of Energy/National Nuclear Security Administration under Award Numbers DENA0003221, DE-NA0000979, DE-NA0002132, and DE-NA00009330.

\section{References}

[1] V. M. Goldschmidt, Skrifter Norske Vitenskapsakad. Oslo. I. Mat.-Naturv. Kl. No. 4 (1937).

[2] K. Lodders, Astrophys. J. 591, 1220 (2003).

[3] E. M. Burbidge, G. R. Burbidge, W. A. Fowler, and F. Hoyle, Rev. Mod. Phys. 29, 547 (1957).

[4] A. G. W. Cameron, Pub. Astron. Soc. Pac. 69, 201 (1957).

[5] M. Arnould, S. Goriely, and K. Takahashi, Phys. Rep. 450, 97 (2007).

[6] H.-T. Janka, Annu. Rev. Nucl. Part. Sci. 62, 407 (2012).

[7] G. Martínez-Pinedo, T. Fischer, A. Lohs, and L. Huther, Phys. Rev. Lett. 109, 251104 (2012).

[8] S. Goriely, J.-L. Sida, J.-F. Lemaitre, S. Panebianco, N. Dubray, S. Hilaire, A. Bauswein, and H.-T. Janka, Phys. Rev. Lett. 111, 242502 (2013).

[9] O. Just, A. Bauswein, R. Ardevol Pulpillo, S. Goriely, H.-T. Janka, Month. Not. Roy. Astron. Soc. 448, 541 (2015).

[10] R. W. P. Drever, F. J. Raab, K. S. Thorne, R. Vogt, and R. Weiss, Laser Interferometer Gravitational-wave Observatory (LIGO) Technical Report, 1989, dcc.ligo.org. 
[11] N. R. Tanvir, A. J. Levan, A. S. Fruchter, J. Hjorth, R. A. Hounsell, K. Wiersema and R. L. Tunnicliffe, Nature 500, 547 (2013).

[12] L.-X. Li and B. Paczynśki, , Astrophys. J. Lett. 507, L59 (1998).

[13] W. Hauser and H. Feshbach, Phys. Rev. 87, 366 (1952).

[14] A. J. Koning and M. C. Duijvestijn, Nucl. Phys. A744, 15 (2004).

[15] Y. Xu and S. Goriely, Phys. Rev. C 86, 045801 (2012).

[16] D. Rochman, S. Goriely, A. J. Koning, and H. Ferroukhi, Phys. Lett. B 764, 109 (2017).

[17] M. R. Mumpower, G. C. McLaughlin, and R. Surman, Phys. Rev. C 86, 035803 (2012).

[18] R. Surman, M. Mumpower, R. Sinclair, K. L. Jones, W. R. Hix, and G. C. McLaughlin, AIP Advances 4, 041008 (2014).

[19] M. R. Mumpower, R. Surman, G. C. McLaughlin, and A. Aprahamian, Prog. Part. Nucl. Phys. 86, 86 (2016).

[20] A. Schiller, L. Bergholt, M. Guttormsen, E. Melby, J. Rekstad, and S. Siem, Nucl. Instrum. Methods Phys. Res. A 447, 498 (2000).

[21] M. Guttormsen, T. S. Tveter, L. Bergholt, F. Ingebretsen, and J. Rekstad, Nucl. Instrum. Methods Phys. Res. A 374, 371 (1996).

[22] M. Guttormsen, T. Ramsøy, and J. Rekstad, Nucl. Instrum. Methods Phys. Res. A 255, 518 (1987).

[23] G. A. Bartholomew, E. D. Earle, A. J. Ferguson, J. W. Knowles, and M. A. Lone, Adv. Nucl. Phys. 7, 229 (1973).

[24] A. C. Larsen et al., Phys. Rev. C 83, 034315 (2011).

[25] A. Voinov et al., Phys. Rev. Lett. 93, 142504 (2004).

[26] A. C. Larsen et al., Phys. Rev. C 76, 044303 (2007).

[27] A. Bürger et al., Phys. Rev. C 85064328 (2012).

[28] M. Guttormsen et al., Phys. Rev. C 71, 044307 (2005).

[29] B. V. Kheswa et al., Phys. Lett. B 744, 268 (2015).

[30] A. Simon et al., Phys. Rev. C 93, 034303 (2016).

[31] M. Wiedeking et al., Phys. Rev. Lett. 108, 162503 (2012).

[32] A. C. Larsen et al., Phys. Rev. Lett. 111, 242504 (2013).

[33] E. Litvinova and N. Belov, Phys. Rev. C 88, 031302(R) (2013).

[34] R. Schwengner, S. Frauendorf, and A. C. Larsen, Phys. Rev. Lett. 111, 232504 (2013).

[35] B. Alex Brown and A. C. Larsen, Phys. Rev. Lett. 113, 252502 (2014).

[36] A. C. Larsen and S. Goriely, Phys. Rev. C 82, 014318 (2010).

[37] H. Utsunomiya et al., Phys. Rev. C 88, 015805 (2013).

[38] T. G. Tornyi et al., Phys. Rev. C 89, 044323 (2014).

[39] T. A. Laplace et al., Phys. Rev. C 93, 014323 (2016).

[40] A. C. Larsen et al., Phys. Rev. C 93, 045810 (2016). 
[41] G. M. Tveten et al., Phys. Rev. C 94, 025804 (2016).

[42] L. Crespo Campo et al., Phys. Rev. C 94, 044321 (2016).

[43] A. J. Koning, S. Hilaire and M. C. Duijvestijn, "TALYS-1.6", Proceedings of the International Conference on Nuclear Data for Science and Technology, April 22-27, 2007, Nice, France, editors O. Bersillon, F. Gunsing, E. Bauge, R. Jacqmin, and S. Leray, EDP Sciences, 2008, p. 211-214.

[44] A. J. Koning and D. Rochman, Nuclear Data Sheets 113, 2841 (2012).

[45] High intensity and Energy-ISOLDE at CERN, HIE-ISOLDE.

[46] S. Siem, M. Wiedeking et al., proposal to the iThemba LABS Program Advisory Committee (2014).

[47] V. W. Ingeberg, Master thesis for the degree M.Sc., The Oslo Method in Inverse Kinematics, Department of Physics, University of Oslo (2016); www.duo.uio.no.

[48] S. Siem, M. Wiedeking et al., proposal to the HIE-ISOLDE Program Advisory Committee (2012); IS559 experiment proposal.

[49] V. Bildstein et al., Eur. Phys. J. A 48, 85 (2012).

[50] P. Reiter et al., Nucl. Phys. A 701, 209 (2002).

[51] A. Spyrou et al., Phys. Rev. Lett. 113, 232502 (2014).

[52] A. Simon, S. Quinn, A. Spyrou et al., Nucl. Instrum. Methods Phys. Res., Sect. A 703, 16 (2013).

[53] D. J. Morrissey, B. M. Sherrill, M. Steiner, A. Stolz, and I. Wiedenhoever, Nucl. Instrum. Methods Phys. Res., Sect. B 204, 90 (2003).

[54] K. Cooper, C. Sumithrarachchi, D. Morrissey, A. Levand, J. Rodriguez, G. Savard, S. Schwarz, and B. Zabransky, Nucl. Instrum. Methods Phys. Res., Sect. A 763, 543 (2014).

[55] S. N. Liddick et al., Phys. Rev. Lett. 116, 242502 (2016).

[56] D. M. Rossi et al., Phys. Rev. Lett. 111, 242503 (2013).

[57] R. H. Cyburt et al., Astrophys. J. Suppl. Ser. 189, 240 (2010); available at https://groups.nscl.msu.edu/jina/reaclib/db/.

[58] Facility for Rare Isotope Beams at Michigan State University, FRIB.

[59] Facility for Antiproton and Ion Research in Europe GmbH, FAIR.

[60] J. Aasi et al., Classical and Quantum Gravity 32, 074001 (2015); see also www.advancedligo.mit.edu

[61] B. P. Abbott et al., Astrophys. J. Lett. 826, L13 (2016). 